\title{
The cumulative effect of inflammation and infection on structural lung disease in early cystic fibrosis
}

\author{
Tim Rosenow (1) ${ }^{1}$, L. Clara Mok (10), Lidija Turkovic ${ }^{1}$, Luke J. Berry', \\ Peter D. Sly ${ }^{2}$, Sarath Ranganathan ${ }^{3,4}$, Harm A.W.M. Tiddens ${ }^{5}$ and \\ Stephen M. Stick ${ }^{1,6,7}$
}

\begin{abstract}
Affiliations: ${ }^{1}$ Telethon Kids Institute, Centre for Child Health Research, University of Western Australia, Subiaco, Australia. ${ }^{2}$ Children's Health and Environment Program, Child Health Research Centre, The University of Queensland, Brisbane, Australia. ${ }^{3}$ Depts of Paediatrics and Respiratory Medicine, Royal Children's Hospital, Melbourne, Australia. 'Infection and Immunity Theme, Murdoch Children's Research Institute, Melbourne, Australia. ${ }^{5}$ Dept of Pulmonology, Sophia Children's Hospital, Erasmus MC, Rotterdam, The Netherlands. 'Dept of Respiratory and Sleep Medicine, Perth Children's Hospital, Nedlands, Australia. ${ }^{7}$ Telethon Kids Respiratory Research Centre, Nedlands, Australia.
\end{abstract}

Correspondence: Tim Rosenow, Telethon Kids Institute, Northern Entrance, Perth Children's Hospital, 15 Hospital Avenue, Nedlands, WA 6009, Australia. E-mail: tim.Rosenowatelethonkids.org.au

@ERSpublications

Pulmonary inflammation and infection have cumulative effects on structural lung disease in young children with CF. These observations underscore the importance of developing anti-inflammatory interventions for use in early life. http://bit.ly/2VRo8qv

Cite this article as: Rosenow T, Mok LC, Turkovic L, et al. The cumulative effect of inflammation and infection on structural lung disease in early cystic fibrosis. Eur Respir J 2019; 54: 1801771 [https://doi.org/ 10.1183/13993003.01771-2018].

\section{ABSTRACT}

Introduction: Pulmonary inflammation and infection are important clinical and prognostic markers of lung disease in cystic fibrosis (CF). However, whether in young children they are transient findings or have cumulative, long-term impacts on respiratory health is largely unknown. We aimed to determine whether their repeated detection has a deleterious effect on structural lung disease.

Methods: All patients aged $<6$ years with annual computed tomography (CT) and bronchoalveolar lavage (BAL) were included. Structural lung disease on CT (\%Disease) was determined using the PRAGMA-CF (Perth-Rotterdam Annotated Grid Morphometric Analysis for CF) method. The number of times free neutrophil elastase (NE) and infection were detected in BAL were counted, to determine cumulative BAL history. Linear mixed model analysis, accounting for repeat visits and adjusted for age, was used to determine associations.

Results: 265 children (683 scans) were included for analysis, with BAL history comprising 1161 visits. \%Disease was significantly associated with the number of prior NE $(0.31,95 \%$ CI $0.09-0.54 ; \mathrm{p}=0.007)$ but not infection $(0.23,95 \% \mathrm{CI}-0.01-0.47 ; \mathrm{p}=0.060)$ detections. Reference equations were determined.

Conclusions: Pulmonary inflammation in surveillance BAL has a cumulative effect on structural lung disease extent, more so than infection. This provides a strong rationale for therapies aimed at reducing inflammation in young children. 


\section{Introduction}

Lung disease in cystic fibrosis (CF) begins early in life and is characterised by an ongoing cycle of pulmonary inflammation, infection and structural lung disease [1,2]. Markers of inflammation, including free neutrophil elastase (NE) and interleukin-8 (IL-8), as well as infection with various pathogenic organisms can be detected in bronchoalveolar lavage (BAL) fluid, even in asymptomatic infants diagnosed by newborn screening [3-6]. In young children, the presence of these markers is associated with lung function at the time of measurement and its subsequent decline, as well as both structural lung disease extent and the onset of bronchiectasis [2, 3, 6-10]. However, the impact of the repeated presence of inflammation and infection on the trajectory of structural lung disease in children $<6$ years of age is not yet well characterised. In particular, it is not known if subclinical (i.e. detected by surveillance rather than upon clinical suspicion) inflammation and infection in this age group are transient, leading to temporary changes in respiratory health, or have cumulative, long-term impacts. As a result there is little consensus on therapeutic strategies for young children. If chronic infection and/or inflammation would have long-term impacts, more aggressive treatment for early CF lung disease would be warranted. Optimising early intervention and monitoring strategies, prior to the development of permanent structural lung disease, is likely to be critical to the long-term preservation of lung function and quality of life of patients with CF. With the recent development of age-appropriate, sensitive outcomes measures based on computed tomography (CT), such as the PRAGMA-CF (Perth-Rotterdam Annotated Grid Morphometric Analysis for CF) method [10], it is now possible to determine how these early measurements relate to the progression of structural lung disease throughout early childhood.

The Australian Respiratory Early Surveillance Team for CF (AREST CF) has collected annual matched CT scans, markers of pulmonary inflammation and pulmonary infection in newborn screened CF patients across two centres since 2005. These data provide an extensive history of inflammation and infection, and are therefore ideally suited to determine the cumulative effect of pulmonary inflammation and infection on structural lung disease progression throughout early childhood. The aim of this study was to evaluate whether repetitive exposure to inflammation and infection leads to worse structural lung disease. We hypothesised that the extent of structural lung disease measured throughout this age period will be associated with cumulative inflammation and infection history, which we defined as the number of times the inflammatory markers (free NE and IL-8) and infection with organisms known to cause lung inflammation [11] were detected in annual surveillance BAL.

\section{Materials and methods \\ Subjects}

Children in this study were participants in the AREST CF early surveillance programme, of whom $>90 \%$ of newborn screened infants attending CF clinics at the Royal Children's Hospital, Melbourne, and Perth Children's Hospital, Perth, participate [12]. The early surveillance programme includes BAL and CT scan performed at 3 months of age and then annually until the age of 6 years. In Melbourne, CT is performed biennially, starting at age 1 year. For the CT analysis, all surveillance visits prior to August 22, 2015 that included a volumetric inspiratory CT scan were included. Volumetric CT scanning was instituted in 2007 (Perth) and 2010 (Melbourne), and hence the CT component of this study covers a period of $\sim 7$ years. All patients are treated with the current standard of care in their respective CF centre, which includes amoxicillin-clavulanate prophylaxis from diagnosis until $\sim 2$ years of age [1].

\section{CT acquisition}

All CT scans were obtained under general anaesthesia with pressure-controlled inspiratory and expiratory series, as previously described $[10,12]$. Inspiratory scans were only analysed if they were performed with a volumetric protocol. Both limited slice and volumetric expiratory scans were analysed, so long as the accompanying inspiratory scan was performed with a volumetric protocol. For volumetric expiratory scans, a 4-5 mm minimum intensity projection filter was applied to aid in trapped air visualisation [10].

\section{CT analysis}

The PRAGMA-CF method was used to determine the extent of structural lung disease (\%Disease), bronchiectasis alone (\%Bronchiectasis) and trapped air (\%TrappedAir) [10]. Scans were de-identified and randomly allocated to one of two trained observers for assessment with PRAGMA-CF. Scans that had been scored by one of the observers for a previous study were not rescored: their original score was used. Intra-observer reliability for PRAGMA-CF outcomes in this age group have previously been published [10]; however, interobserver variability was determined from a random subset of 30 scans. 
Inflammation and infection

History of Inflammation and infection was determined from all annual surveillance visits, regardless of whether a CT was performed. Treatment with antibiotic therapy is not routinely ceased prior to CT or BAL. The presence of detectable free NE and IL-8 concentration were measured from BAL, as previously described [12]. A significant pro-inflammatory infection was defined as detection in BAL fluid of $\geqslant 10^{4} \mathrm{CFU} \cdot \mathrm{mL}^{-1}$ of one of the following pathogens previously reported to be associated with pulmonary inflammation: Pseudomonas aeruginosa, Staphylococcus aureus, Haemophilus influenzae, Streptococcus pneumoniae and Aspergillus species [11]. To determine cumulative inflammation and infection history, we calculated the number of prior annual surveillance visits with a positive NE detection, a positive IL-8 detection and significant pro-inflammatory infection (multiple organisms not counted twice), separately.

\section{Statistical analysis}

All statistical analyses were performed using $\mathrm{R}$ version 3.2.5 (R Foundation for Statistical Computing, Vienna, Austria) with the packages lme4 and lmerTest [13-15]. The concordance correlation coefficient was determined to assess interobserver variability. Linear mixed model analysis was used to determine the relationships between $\mathrm{CT}$ and BAL outcomes, adjusting for age and with random intercepts and slopes (representing patient ID) fitted, to represent repeated visits. Both cross-sectional and interval (change in PRAGMA-CF outcomes over year: $\Delta \%$ Disease, $\Delta \%$ Bronchiectasis and $\Delta \%$ TrappedAir) analyses were performed. In addition, the cumulative effects of inflammation and infection on structural lung disease were assessed by determining the associations between PRAGMA-CF for a given visit and the cumulative $\mathrm{BAL}$ results from all prior visits. Finally, CF-specific reference equations (to evaluate the natural progression of lung disease in this population) were determined using age, sex and genotype severity as predictors. Patients with unknown genotype severity were excluded from this reference population.

\section{Results}

\section{Study population}

265 patients were included in this study, with 683 scans available for analysis at a mean \pm SD age of $3.3 \pm 1.8$ years. Of these scans, 664 had matched inflammation data and 620 had matched infection data. Interval CT data were available in 127 patients (342 scans). There were 1161 BALs (with or without matched CT) to determine cumulative inflammatory/infective history in these patients. The most common causes for missing data were insufficient BAL fluid collected for analysis, equipment failure or sample contamination. CT scans were only performed biennially in Melbourne, so 1-year interval data were only available in the Perth cohort (127 patients, 342 scan pairs). There were no significant differences between Perth and Melbourne in PRAGMA-CF outcomes, presence of free NE activity or IL-8 concentration; however, the presence of pro-inflammatory pathogens was significantly higher in Melbourne (mixed model coefficient $0.35,95 \%$ CI $0.26-0.44 ; \mathrm{p}<0.001)$. Demographic and key cohort outcomes are presented in table 1.

\section{Observer reliability}

Prior to analysis, the interobserver reliability between the two PRAGMA-CF scorers was determined by duplicate scoring, in a random order, 30 randomly selected scans. The concordance correlation coefficients for each component were: \%Disease 0.804 (95\% CI $0.695-0.877)$, \%Bronchiectasis $0.629 \quad(95 \%$ CI 0.521-0.718) and \%TrappedAir 0.889 (95\% CI 0.787-0.944).

\section{Cross-sectional relationships between CT outcomes and BAL}

All PRAGMA-CF outcomes were significantly associated with age. In addition, all PRAGMA-CF outcomes were significantly associated with inflammatory markers, i.e. IL-8 concentration and NE activity presence. $\%$ Disease and \%TrappedAir, but not \%Bronchiectasis, were associated with the presence of a pro-inflammatory infection. Cross-sectional correlation data are presented in table 2.

\section{Relationships between interval CT outcomes and BAL}

To determine the short-term effect of inflammation on structural lung disease progression, we compared the change in PRAGMA-CF outcomes over 1 year to the presence of NE activity and concentration of IL-8 at time-point 2, adjusted for age and for baseline PRAGMA-CF score. The same analysis was performed for pro-inflammatory infections. Data were available for 340 scan pairs. For \%Disease and \%TrappedAir, the progression over 1 year was associated with the presence of NE activity, concentration of IL- 8 and presence of pro-inflammatory infection. Only NE activity was associated with \%Bronchiectasis (table 3).

\section{Relationships between CT and BAL history}

To determine the cumulative effects of inflammation on structural lung disease, we separately compared PRAGMA-CF outcomes to the number of times NE activity and IL-8 were detected in visits prior to the CT scan, adjusted for age. The same analysis was performed for pro-inflammatory infections. The number 


\begin{tabular}{|c|c|}
\hline \multicolumn{2}{|l|}{ Clinical characteristics } \\
\hline CT scans (patients) & $683(265)$ \\
\hline Total BALs & 1161 \\
\hline Age at $\mathrm{CT}$ scan years & $3.30 \pm 1.78$ \\
\hline \multicolumn{2}{|l|}{ Centre } \\
\hline Perth & 154 \\
\hline Melbourne & 111 \\
\hline \multicolumn{2}{|l|}{ Sex } \\
\hline Male & 135 \\
\hline Female & 130 \\
\hline Severe genotype $e^{\#}$ & $192 / 210(91)$ \\
\hline Pancreatic insufficient ${ }^{\#}$ & $199 / 243(82)$ \\
\hline \multicolumn{2}{|l|}{ CT measures } \\
\hline$\%$ Disease & $3.19 \pm 2.29$ \\
\hline$\%$ Bronchiectasis & $0.37 \pm 1.21$ \\
\hline \%TrappedAir & $4.30 \pm 7.39$ \\
\hline Interval scan pairs" & 342 \\
\hline$\Delta \%$ Disease & $0.82 \pm 2.35$ \\
\hline$\Delta \%$ Bronchiectasis ${ }^{\pi}$ & $0.18 \pm 1.22$ \\
\hline$\Delta \%$ TrappedAir ${ }^{\uparrow}$ & $0.79 \pm 8.22$ \\
\hline \multicolumn{2}{|l|}{ BAL measures } \\
\hline Free NE activity present $\#$ & $135 / 664(20)$ \\
\hline IL-8 present ${ }^{\#}$ & $632 / 663(95)$ \\
\hline IL-8 concentration ${ }^{\#} \mathrm{ng} \cdot \mathrm{mL}^{-1}$ & $2.87 \pm 7.69$ \\
\hline Pro-inflammatory infection present ${ }^{\#}$ & $158 / 620(25)$ \\
\hline Previous NE activity detections ${ }^{+}$ & $0.55 \pm 0.93$ \\
\hline Previous IL-8 detections ${ }^{+}$ & $2.45 \pm 1.7$ \\
\hline Previous infection detections ${ }^{+}$ & $0.57 \pm 0.86$ \\
\hline
\end{tabular}

Data are presented as $\mathrm{n}$, mean \pm SD or fraction (\%), unless otherwise stated. BAL: bronchoalveolar lavage; CT: computed tomography; NE: neutrophil elastase; IL-8: interleukin-8. ${ }^{\#}$ : data for some patients and/or visits are unknown or not recorded; " : changes in CT measures over time are from all patients with two CT scans, 1 year apart; ${ }^{+}$: number of NE detections, IL-8 detections, pro-inflammatory infection detections and organisms recorded from annual visits performed prior to the CT scan.

of NE activity detections was significantly associated with all CT outcomes. Neither the number of IL-8 detections nor infections was associated with CT outcomes (table 2). The association between age and $\%$ Disease, coloured according to the number of detections of NE and infection, are shown in figure 1 .

\section{Reference equations}

Reference equations for PRAGMA-CF are presented in table 4. Patients with unknown genotype severity (55 out of 265) were not included in this analysis. Centre (Perth versus Melbourne) was not a significant predictor and its inclusion in the model had little effect on the coefficients, so it was not included in the final prediction equations. Reference equation coefficients are presented in table 4.

TABLE 2 Associations between computed tomography (CT) and bronchoalveolar lavage (BAL) measures

\begin{tabular}{|c|c|c|c|c|c|c|}
\hline & \multicolumn{2}{|l|}{ \%Disease } & \multicolumn{2}{|c|}{$\%$ Bronchiectasis } & \multicolumn{2}{|c|}{ \%TrappedAir } \\
\hline Age & $0.54(0.46-0.63)$ & $<0.001$ & $0.14(0.10-0.19)$ & $<0.001$ & $0.69(0.41-0.97)$ & $<0.001$ \\
\hline IL-8 (log) & $0.30(0.20-0.40)$ & $<0.001$ & $0.05(0.00-0.09)$ & 0.035 & $0.94(0.61-1.27)$ & $<0.001$ \\
\hline Infection" & $0.65(0.28-1.01)$ & $<0.001$ & $0.09(-0.08-0.26)$ & 0.282 & $2.84(1.57-4.12)$ & $<0.001$ \\
\hline Previous NE activity detections ${ }^{\pi}$ & $0.31(0.09-0.54)$ & 0.007 & $0.13(0.01-0.25)$ & 0.029 & $1.45(0.69-2.20)$ & $<0.001$ \\
\hline
\end{tabular}

Linear mixed model data between CT and BAL measures of infection and inflammation. Age is included as a fixed effect in each model, and random intercepts and slopes for each patient. NE: neutrophil elastase; IL-8: interleukin-8. \#: detection of significant colonisation of a pro-inflammatory pathogen; ": number of detections of NE and any pro-inflammatory infection detections in annual surveillance BALs performed prior to the CT scan. 
TABLE 3 Associations between 1-year computed tomography (CT) interval data and bronchoalveolar lavage (BAL) measures

\begin{tabular}{|c|c|c|c|c|c|c|}
\hline & \multicolumn{2}{|l|}{$\Delta \%$ Disease } & \multicolumn{2}{|c|}{$\Delta \%$ Bronchiectasis } & \multicolumn{2}{|l|}{$\Delta \%$ TrappedAir } \\
\hline & Coefficient $(95 \% \mathrm{Cl})$ & p-value & Coefficient $(95 \% \mathrm{Cl})$ & p-value & Coefficient $(95 \% \mathrm{Cl})$ & p-value \\
\hline NE activity & 1.80 (1.22-2.39) & $<0.001$ & $0.62(0.33-0.91)$ & $<0.001$ & $6.84(4.83-8.84)$ & $<0.001$ \\
\hline IL-8 (log) & $0.38(0.23-0.54)$ & $<0.001$ & $0.04(-0.04-0.11)$ & 0.342 & $1.25(0.69-1.80)$ & $<0.001$ \\
\hline Infection" & $0.90(0.32-1.49)$ & 0.003 & $0.04(-0.24-0.32)$ & 0.777 & $3.25(1.12-5.38)$ & 0.003 \\
\hline
\end{tabular}

Linear mixed model data between change in CT outcome over 1 year and BAL measures of infection and inflammation at the follow-up scan. Both age and baseline CT measure are included as fixed effects in each model, and random intercepts and slopes for each patient. NE: neutrophil elastase; IL-8: interleukin-8. " : detection of significant colonisation of a pro-inflammatory pathogen.

\section{Discussion}

These data provide unique insight regarding the cumulative effects of inflammation and infection on structural lung disease in young children with CF. We demonstrated that not only is structural lung disease associated with the detection of inflammation and infection, but that repeated detection of free NE activity is associated with worse CT outcomes. This implies that inflammation that exceeds anti-inflammatory defences in the lungs in the first 6 years of life has a cumulative, ongoing effect on lung disease progression, plays a substantial role in the pathogenesis of CF-related structural lung disease and that preventing its presence has the potential to reduce the long-term impact of CF lung disease. These data present a strong case for trials of prophylactic anti-inflammatory therapy targeted at reducing neutrophilic inflammation in this age group, with the aim to prevent structural lung disease at the age of 6 years.

\section{Structural lung disease}

The variability in structural lung disease extent between patients seen in this study is consistent with the heterogeneous nature of CF lung disease. Nevertheless, as expected, an overall progression over time was observed, with \%Disease increasing by around 0.5 per year. Furthermore, to this end we have presented z-score equations for all PRAGMA-CF outcomes. Although this provides a useful estimate for future studies in this age group and may inform clinical trial design, all children in this study were part of a standardised, extensive early surveillance programme. Therefore, these results may not be directly comparable to other centres with different monitoring and treatment regiments.

The repeatability of \%Bronchiectasis in this study was somewhat lower than originally reported in the PRAGMA-CF publication [10]. This may be the result of the longer time period over which CT scans
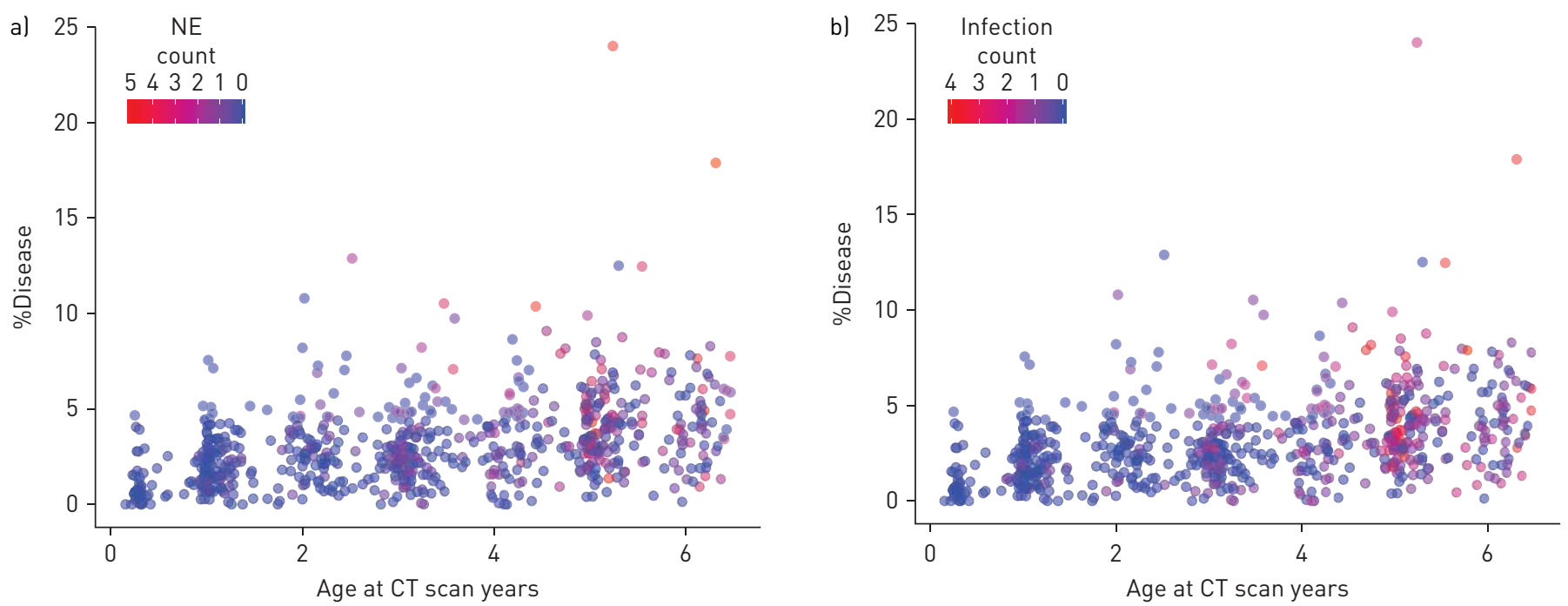

FIGURE 1 The association between the PRAGMA-CF (Perth-Rotterdam Annotated Grid Morphometric Analysis for CF) measure \%Disease and age. a) Colour scale indicates the number of times free neutrophil elastase activity was detected in annual surveillance bronchoalveolar lavage (BAL) prior to the computed tomography (CT) date. b) Colour scale indicates the number of times a pro-inflammatory infection was detected in annual surveillance BAL prior to the CT date. 
TABLE 4 Prediction equation coefficients for PRAGMA-CF (Perth-Rotterdam Annotated Grid Morphometric Analysis for Cystic Fibrosis) outcomes

\begin{tabular}{lccccc} 
Outcome & Age years & $\begin{array}{c}\text { Genotype } \\
\text { (0=mild, } 1=\text { severe) }\end{array}$ & $\begin{array}{c}\text { Sex } \\
\text { (0=male, 1=female) }\end{array}$ & Intercept & Residual SD \\
\hline \%Disease & 0.564 & 0.878 & 0.441 & 0.244 & 1.421 \\
\%Bronchiectasis & 0.155 & 0.267 & 0.329 & -0.581 & 0.838 \\
\%TrappedAir & 0.745 & 2.052 & 1.864 & -1.114 & 4.972
\end{tabular}

Prediction equations for PRAGMA-CF computed tomography outcomes for children aged $<6$ years, in the Australian Respiratory Early Surveillance Team for CF cohort. The reference value for a given patient for each outcome can be calculated by summing the predictors multiplied by their listed coefficient and adding the intercept. The z-score can be calculated by subtracting the predicted value from the measured value and dividing by the residual standard deviation.

were collected, and thus a greater variety in CT settings and image quality. Because bronchiectasis is rare in young children compared with other abnormalities such as bronchial wall thickening, its extent tends to be quite low (in this study $0.37 \%$ of the lung) and thus it is subject to greater variability. In young children with small airways, determining the airway/vessel ratio (and thus bronchiectasis) can be a matter of one or two pixels on the CT scan. Nevertheless, because the scans were allocated randomly and scorers were blinded to patient details, clinical status or BAL results, variability in \%Bronchiectasis is likely to add noise to our measurements but not bias the results.

\section{Relationships between CT and inflammation/infection}

As expected, we found that all CT outcomes were associated with detectable free NE activity, IL-8 concentration and infection presence in cross-sectional analyses, other than between \%Bronchiectasis and infection. We also found that CT outcomes increased in the 1-year interval prior to the detection of NE activity or infection. These results are consistent with previous studies relating CT to lower airway samples $[2,10,16,17]$. The most important finding from this study was that structural lung disease extent was associated with the number of previous visits with BAL-detected NE activity, but not pulmonary infection. There is currently no consensus on whether pulmonary inflammation in young children is a transient effect with little long-term consequence or a clinically important finding that warrants treatment. The results of this study seem to suggest the latter, although prospective clinical trials are needed to confirm whether structural lung disease can be prevented with targeted interventions.

\section{Comparison between inflammation and infection}

The results of this study show that inflammation, particularly free NE activity, has a stronger correlation with and larger effect on structural lung disease than infection. Therefore, treating lung inflammation has additional potential for reducing structural lung disease compared with using anti-infective therapies alone. Despite this, detection of NE activity or another marker of lung inflammation is rarely acted upon, whereas pulmonary infection with, for example, $P$. aeruginosa is often considered to be a clinically significant finding necessitating eradication therapy $[18,19]$. Numerous studies have shown that eradication of this organism when first detected leads to delayed onset of chronic infection and improved clinical outcomes throughout childhood [20-24], and there is mounting evidence that eradication of other organisms such as S. aureus may also be indicated [25]. However, aggressive Pseudomonas eradication strategies fail to prevent the high prevalence of bronchiectasis seen at 5 years of age [26], suggesting that chronic inflammation, regardless of the triggers, is a critical factor in the development of structural lung damage [27]. Prophylactic anti-inflammatory therapy is rarely included in routine management early in life, although in children aged $>6$ years and in adults there is evidence for clinical benefits and its use is included in some clinical guidelines [28, 29]. However, although the side-effects profile of ibuprofen limits its use in newborns and infants, there are significant opportunities for repurposing a number of alternative anti-inflammatory molecules. The prevention of bronchiectasis in infants with CF study (COMBAT-CF; ClinicalTrials.gov identifier NCT01270074) of prophylactic azithromycin from diagnosis provides a template for studies of repurposed molecules to prevent lung disease. Such an approach could result in the relatively expeditious implementation of new anti-inflammatory interventions for young children with $\mathrm{CF}$.

\section{Reference equations}

The reference equations in this study are CF specific, in that they capture the "usual" trajectory of lung disease in CF in a population undergoing "standard" treatment. These values may add clarity when comparing consecutive CT scans, to differentiate between natural disease progression and a potentially clinically significant 
change. Additionally, these values may aid in clinical trial design, e.g. when determining a clinically relevant treatment effect. However, it is well recognised that there exists significant variability in patient outcomes between countries, centres and even individual clinicians. The equations presented are only directly applicable to the centres in this study; other centres should use them only as a guide, ideally developing their own using the methods in this study. With the advent of CF transmembrane conductance regulator modulators and other disease-modifying medications, none of which were available for young children during the period of this study, consideration of their use should be taken into account when using these reference ranges.

\section{Limitations}

Although both NE activity and IL-8 are important indicators, their absence does not imply an absence of pulmonary inflammation. In addition, IL- 8 was present in the majority of BALs and thus the number of times it is detected is not a particularly informative measure. The binary classification of NE activity into present/absent may mask the effects of inflammation severity on structural lung disease. A comprehensive survey of biochemical measures and cell counts would provide a more holistic view of lung inflammation. Furthermore, BAL samples are taken from individual lobes and may not be representative of the entire lung. Nevertheless, free NE activity in particular is a known risk factor for structural lung disease in early life CF [2] and its repeated detection from BAL provides a simple yet meaningful summary of inflammatory history.

Infection was detected using culture methods, as per routine clinical practice. Because bacterial cultures are not as sensitive to detect pathological organisms as other methods, e.g. gene sequencing or mass spectrometry [30], it is likely that the bacterial infection was underestimated using this study. The prohibitive cost and lack of availability of these techniques preclude their use in this retrospective analysis. However, the clinical impact of these infections that are unable to be cultured is not yet known, whereas culture of bacteria from BAL is a known prognostic indicator, and thus these results still provide a compelling argument for the importance of early life infection and inflammation in the pathogenesis of structural lung disease.

Our data were acquired as part of annual surveillance snapshots and thus may not accurately reflect the course of CF lung disease in the intervening year between visits. However, the strength of this approach is that the full spectrum of CF lung disease is represented, unlike studies recruited based upon episodes of acute disease. Because visits were scheduled at regular intervals while the children were clinically well, selection bias is unlikely to affect the results presented.

Due to differences in clinical protocol, CT scans were only performed every second year in Melbourne, but annually in Perth. As a result, the associations reported will be more heavily weighted towards data from the Perth centre, with the 1-year interval data exclusively from Perth. Both centres performed BAL annually, so the cumulative inflammation and infection results are not affected, and therefore although there is a Perth bias, this does not affect the interpretation of our results.

Finally, there were a number of visits where inflammation and/or infection measurements were not available, either through failure to collect adequate sample or nonattendance at the scheduled BAL. Missed visits will result in an underestimation of inflammatory/infective history, particularly if the visit was missed due to the patient being too unwell. However, the number of missed visits was small and unlikely to influence the results substantially.

\section{Conclusions}

We have shown that repeated detection of neutrophilic inflammation plays an important and ongoing role in the trajectory of structural lung disease throughout the first 6 years of life. Although infection is generally treated aggressively, inflammation is not routinely measured nor acted upon. These data present compelling evidence that pulmonary inflammation, particularly NE activity, throughout early life is a key prognostic indicator. Therefore, early-life anti-inflammatory prophylaxis could be a useful strategy to improve long-term outcomes and further trials aimed at preventing lung disease are urgently needed in this young age group.

Acknowledgements: The authors wish to thank Australia's National Health and Medical Research Council and the US Cystic Fibrosis Foundation for funding this study. In addition, we wish to thank the Dept of Diagnostic Imaging at Perth Children's Hospital (Perth, Australia) for their assistance in obtaining and retrieving CT scans.

Author contributions: Literature search: T. Rosenow and S.M. Stick. Figure: T. Rosenow and L. Turkovic. Study design: all authors equally. Data collection: T. Rosenow, L.C. Mok and L.J. Berry. Data analysis: T. Rosenow and L. Turkovic. Data interpretation: all authors equally. Manuscript writing: all authors equally

Conflict of interest: T. Rosenow reports grants from the National Health and Medical Research Council, during the conduct of the study, and in addition, has a patent PCT/AU2016/000079 pending. L.C. Mok has nothing to disclose. L. Turkovic has nothing to disclose. L.J. Berry has nothing to disclose. P.D. Sly has nothing to disclose. S. Ranganathan has nothing to disclose. H.A.W.M. Tiddens reports other funding from Roche and Novartis, and grants from CFF, Vertex, Gilead and Chiesi, outside the submitted work; in addition, has a patent Vectura licensed and a patent PRAGMA-CF scoring system 
issued; and is also head of the Erasmus MC-Sophia Children's Hospital core laboratory lung analysis. S.M. Stick reports receiving grants from the National Health and Medical Research Council, during the conduct of the study.

Support statement: This study was supported by Australian National Health and Medical Research Council grants APP102055 and APP1000896, and US Cystic Fibrosis Foundation grants SLYA04 and STICKA010. Funding information for this article has been deposited with the Crossref Funder Registry.

\section{References}

1 Sly PD, Brennan S, Gangell C, et al. Lung disease at diagnosis in infants with cystic fibrosis detected by newborn screening. Am J Respir Crit Care Med 2009; 180: 146-152.

2 Sly PD, Gangell CL, Chen L, et al. Risk factors for bronchiectasis in children with cystic fibrosis. $N$ Engl J Med 2013; 368: 1963-1970.

3 Dakin CJ, Numa $\mathrm{AH}$, Wang $\mathrm{H}$, et al. Inflammation, infection, and pulmonary function in infants and young children with cystic fibrosis. Am J Respir Crit Care Med 2002; 165: 904-910.

4 Armstrong DS, Hook SM, Jamsen KM, et al. Lower airway inflammation in infants with cystic fibrosis detected by newborn screening. Pediatr Pulmonol 2005; 40: 500-510.

5 Brennan S, Gangell C, Wainwright C, et al. Disease surveillance using bronchoalveolar lavage. Paediatr Respir Rev 2008; 9: 151-159.

6 Belessis Y, Dixon B, Hawkins G, et al. Early cystic fibrosis lung disease detected by bronchoalveolar lavage and lung clearance index. Am J Respir Crit Care Med 2012; 185: 862-873.

$7 \quad$ Pillarisetti N, Williamson E, Linnane B, et al. Infection, inflammation, and lung function decline in infants with cystic fibrosis. Am J Respir Crit Care Med 2011; 184: 75-81.

8 Ramsey KA, Ranganathan S, Park J, et al. Early respiratory infection is associated with reduced spirometry in children with cystic fibrosis. Am J Respir Crit Care Med 2014; 190: 1111-1116.

9 Simpson SJ, Ranganathan S, Park J, et al. Progressive ventilation inhomogeneity in infants with cystic fibrosis after pulmonary infection. Eur Respir J 2015; 46: 1680-1690.

10 Rosenow T, Oudraad MCJ, Murray CP, et al. PRAGMA-CF. A quantitative structural lung disease computed tomography outcome in young children with cystic fibrosis. Am J Respir Crit Care Med 2015; 191: 1158-1165.

11 Gangell C, Gard S, Douglas T, et al. Inflammatory responses to individual microorganisms in the lungs of children with cystic fibrosis. Clin Infect Dis 2011; 53: 425-432.

12 Stick SM, Brennan S, Murray C, et al. Bronchiectasis in infants and preschool children diagnosed with cystic fibrosis after newborn screening. J Pediatr 2009; 155: 623-628.

13 Bates D, Mächler M, Bolker B, et al. Fitting linear mixed-effects models using lme4. J Stat Softw 2015; 67: 48.

14 Kuznetsova A, Brockhoff PB, Christensen RHB. lmerTest: tests in linear mixed effects models. J Stat Softw 2017; 82: 13.

15 R Core Team. R: A Language and Environment for Statistical Computing. Vienna, R Foundation for Statistical Computing, 2014.

16 Davis SD, Fordham LA, Brody AS, et al. Computed tomography reflects lower airway inflammation and tracks changes in early cystic fibrosis. Am J Respir Crit Care Med 2007; 175: 943-950.

17 Mott LS, Park J, Murray CP, et al. Progression of early structural lung disease in young children with cystic fibrosis assessed using CT. Thorax 2012; 67: 509-516.

18 Douglas TA, Brennan S, Gard S, et al. Acquisition and eradication of P. aeruginosa in young children with cystic fibrosis. Eur Respir J 2009; 33: 305-311.

19 Mogayzel PJ Jr, Naureckas ET, Robinson KA, et al. Cystic Fibrosis Foundation pulmonary guideline. Pharmacologic approaches to prevention and eradication of initial Pseudomonas aeruginosa infection. Ann Am Thorac Soc 2014; 11: $1640-1650$

20 Aebi C, Bracher R, Liechti-Gallati S, et al. The age at onset of chronic Pseudomonas aeruginosa colonization in cystic fibrosis - prognostic significance. Eur J Pediatr 1995; 154: 9 Suppl. 4, S69-S73.

21 Gibson RL, Burns JL, Ramsey BW. Pathophysiology and management of pulmonary infections in cystic fibrosis. Am J Respir Crit Care Med 2003; 168: 918-951.

22 Hoiby N, Frederiksen B, Pressler T. Eradication of early Pseudomonas aeruginosa infection. J Cyst Fibros 2005; 4: Suppl. 2, 49-54.

23 Taccetti G, Campana S, Festini F, et al. Early eradication therapy against Pseudomonas aeruginosa in cystic fibrosis patients. Eur Respir J 2005; 26: 458-461.

24 Valerius NH, Koch C, Hoiby N. Prevention of chronic Pseudomonas aeruginosa colonisation in cystic fibrosis by early treatment. Lancet 1991; 338: 725-726.

25 Goss CH, Muhlebach MS. Review: Staphylococcus aureus and MRSA in cystic fibrosis. J Cyst Fibros 2011; 10: $298-306$.

26 Wainwright CE, Vidmar S, Armstrong DS, et al. Effect of bronchoalveolar lavage-directed therapy on Pseudomonas aeruginosa infection and structural lung injury in children with cystic fibrosis: a randomized trial. JAMA 2011; 306: 163-171.

27 Montgomery ST, Mall MA, Kicic A, et al. Hypoxia and sterile inflammation in cystic fibrosis airways: mechanisms and potential therapies. Eur Respir J 2017; 49: 1600903.

28 Mogayzel PJ Jr, Naureckas ET, Robinson KA, et al. Cystic fibrosis pulmonary guidelines. Chronic medications for maintenance of lung health. Am J Respir Crit Care Med 2013; 187: 680-689.

29 Konstan MW, Schluchter MD, Xue W, et al. Clinical use of ibuprofen is associated with slower FEV $\mathrm{F}_{1}$ decline in children with cystic fibrosis. Am J Respir Crit Care Med 2007; 176: 1084-1089.

30 Caliendo AM, Gilbert DN, Ginocchio CC, et al. Better tests, better care: improved diagnostics for infectious diseases. Clin Infect Dis 2013; 57: Suppl. 3, S139-S170. 